\title{
Computertomographie-Scanner in der Abklärung der koronaren Herzerkrankung
}

\begin{abstract}
Expertenrat des Swiss Medical Board*: Eva Cignacco $^{a}$, Peter Jüni ${ }^{b}$, Peter Meier-Abt ${ }^{c}$, Urs Metzger , Nikola BillerAndorno ${ }^{e}$, Stefan Felderf, Brigitte Tagg

a Institut für Pflegewissenschaft, Universität Basel

b Professor für Klinische Epidemiologie, Universität Bern

c em. Professor für Klinische

Wie gut ist die diagnostische Aussagekraft einer kardialen Computertomographie mit Geräten der neuen Generation (New Generation Cardiac Computed Tomography/ NGCCT) im Vergleich zur üblichen invasiven Koronarangiographie? Dieser Frage ist das Fachgremium Swiss Medical Board in dem soeben veröffentlichten Bericht nachgegangen. Dabei stützt es sich für die Ermittlung der klinischen Evidenz auf drei Dokumente des National Institute of Health and Clinical Excellence (NICE) und kommt zum Schluss, dass bei gewissen Patientengruppen eine kardiale Computertomographie zur Diagnose einer koronaren Herzkrankheit indiziert ist.
\end{abstract} Pharmakologie und Toxikologie, Universität Zürich

d Professor em. Chefarzt Chirurgie, Zürich

e Professorin für Biomedizinische Ethik, Universität Zürich

f Professor für Health Economics, Universität Basel

g Professorin für Straf-, Strafprozess- und Medizinrecht, Universität Zürich

\footnotetext{
* Organisation und personelle Besetzung unter www.medical-board.ch/ index.php?id=818
}

\section{Korrespondenz:}

Susanna Marti Calmell

Sekretariat Trägerschaft

Swiss Medical Board

Obstgartenstrasse 21

CH-8090 Zürich

Tel. 0432592479

info[at]medical-board

www.swissmedicalboard.ch

\section{Kurzfassung aus dem Originalbericht}

Als koronare Herzerkrankung (KHK) werden krankhafte Veränderungen der Arterien bezeichnet, die den Herzmuskel mit Blut versorgen (Herzkranzgefässe oder Koronararterien). Hierbei kommt es zu einer zunehmenden Verminderung des Gefässquerschnitts, welche mittelbar zu akuten, lebensbedrohlichen Komplikationen (z.B. Herzinfarkt) führen kann. In der Schweiz sterben pro Jahr 8800 Personen an einer koronaren Herzerkrankung. Dies entspricht 14,5\% der Gesamtzahl der Todesfälle. Zum Nachweis einer KHK stehen unter anderem zwei bildgebende Verfahren zur Verfügung: die kardiale Computertomographie und die invasive Koronarangiographie (IKA).

Im vorliegenden Bericht des Swiss Medical Board wird die Frage bearbeitet, wie gut die diagnostische Aussagekraft einer kardialen Computertomographie mit Geräten der neuen Generation (New Generation Cardiac Computed Tomography/NGCCT) im Vergleich zur IKA (Goldstandard) ist. Zusätzlich werden potentiell negative Wirkungen der beiden Verfahren und das Kosten-Wirksamkeits-Verhältnis betrachtet. Grundlage für den vorliegenden Bericht sind drei Dokumente des National Institute of Health and Clinical Excellence (NICE) in Grossbritannien sowie ergänzende Überlegungen des Swiss Medical Board.

Auf Basis dieser Grundlagen kann gefolgert werden, dass die kardiale Computertomographie die IKA zur Diagnose einer vermuteten KHK bei einigen Personengruppen ohne Nachteil substituieren kann. Dies ist abhängig von der sogenannten Vortestwahrscheinlichkeit, d.h. der ohne nähere Abklärung eingeschätzten Wahrscheinlichkeit tatsächlich an einer KHK erkrankt zu sein. Das Kosten-Wirksamkeits-Verhältnis der IKA ist bei einigen Personengruppen ver- gleichsweise ungünstig. Die Erkenntnisse aus der vorliegenden Untersuchung führen zu folgenden Empfehlungen:

- Vor der Durchführung eines bildgebenden Verfahrens bei Patienten mit einer vermuteten KHK ist vorab die Vortestwahrscheinlichkeit sorgfältig abzuschätzen.

- Bei Vorliegen einer geringen Vortestwahrscheinlichkeit (typischerweise unter 10\%) ist die Durchführung eines bildgebenden Verfahrens nicht indiziert.

- Bei Personen mit einer geschätzten Vortestwahrscheinlichkeit von ca. 10 bis $30 \%$ soll für die Diagnose eine kardiale Computertomographie mit Geräten der neueren Generation (NGCCT) durchgeführt werden, sofern ein bildgebendes Verfahren überhaupt erforderlich ist. Die Durchführung einer IKA a priori ist bei dieser Personengruppe nicht indiziert. Ob bei positiven Befunden anschliessend eine IKA durchgeführt werden soll, hängt vom CT-Befund und vom individuellen Fall ab.

- Bei Vorliegen einer höheren Vortestwahrscheinlichkeit (typischerweise ca. 30 bis 50\%) soll als erstes bildgebendes Verfahren eine kardiale Computertomographie durchgeführt werden. Bei positiven NGCCT-Befunden sollte gegebenenfalls mit einer IKA weiter abgeklärt und eventuell therapiert werden.

- Die Leistungserbringer haben die Patientinnen und Patienten über die verschiedenen Verfahren aufzuklären, auch wenn eines in der eigenen Institution nicht angeboten werden kann.

\section{Themen zur Bearbeitung durch das Fach- gremium im Jahr 2013 \\ Für das Jahr 2013 wurden nach dem üblichen Verfahren (Stakeholder-Input, Beurteilung nach Priorisierungsraster, Entscheid Trägerschaft) vorerst zwei Themen für die Erstellung von HTA- Berichten ausgewählt: "Systematisches Mammo- graphie-Screening» und «Therapie mit Statinen zur primären Prävention von kardiovaskulären Erkran- kungen».}

Nähere Informationen unter: www.swissmedicalboard.ch 